\title{
Pharmacological Applications of Isorhamnetin: A Short Review
}

\author{
S Kandakumar \\ Research Scholar, Department of Biochemistry, \\ Periyar University, Salem, Tamil Nadu, India
}

\author{
Dr V Manju \\ Assistant Professor, Department of Biochemistry, \\ Periyar University, Salem, Tamil Nadu, India
}

\begin{abstract}
Isorhamnetin (30-Methoxy-3, 40, 5, 7tetrahydroxyflavone) is a flavonol, and it belongs to a group of plant secondary metabolites known as flavonoids. It is present in man's daily diet and is known for its biological activities such as antioxidant, antiviral, anticancer, antimicrobial, and antiinflammatory effects. Isorhamnetin has been reported for its antioxidant and antiviral applications hence it is not only used as such, but it also has various derivatized forms which has potentials for development into drugs for the treatment of diseases caused by oxidative stress and lethal viruses. The present review reflects the pharmacological importance of isorhamnetin.
\end{abstract}

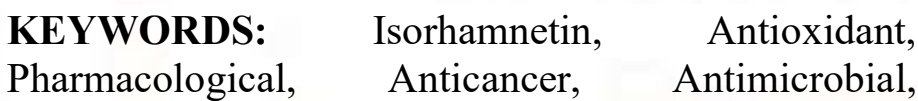
Antiviral, Hepatoprotective

\section{INTRODUCTION}

Flavonoids are natural polyphenolic compounds containing two benzene rings linked with heterocyclic pyrone ring, recently attention have been focused on the antioxidative activities of several plant phenolics, especially flavonoids, because they inhibit lipid peroxidation, and the significance of potential protective properties of flavonoids present in vegetables and fruits has become an important matter $[1,2]$. They are a large group of secondary plant metabolites and also usual constituents of the human diet comprising in excess of six thousands identified compounds are characterized from a variety of plant sources. Until now, a huge number of biological and pharmacological effects have been ascribed to flavonoids, including anti-allergic, anti-inflammatory, antioxidant, antitumor, antiviral, and antimicrobial activities ${ }^{[3-5]}$. Flavonoids are mainly present in tea, red wine, aromatic plants, medical herbs, fruits, and vegetables ${ }^{[6]}$. Epidemiological studies point out that use of foods rich in flavonoids may reduce the incidence of existence diseases ${ }^{[7,8]}$.
The flavonoid isorhamnetin also called as 3'-methoxy3, 4', 5, 7-tetrahydroxyflavone (Fig 1), is the metabolite of quercetin and it is naturally occurring O-methylated flavonol that is plentiful in apples, blackberries, cherries, and pears ${ }^{[9-13]}$. It is also present in medicinal herbs and plants, such as the sea buckthorn (Hippophaer hamnoides L.) and water dropwort (Oenanthe javanica), which are commonly used in the Greek and Danish traditional medicines for the prevention and treatment of a range of diseases $\left[\begin{array}{ll}14 & 15\end{array}\right]$. Therefore, this review focuses on the pharmacological importance of isorhamnetin.

\section{PHARMACOLOGICAL IMPORTANCE OF ISORHAMNETIN}

The drug isorhamnetin is a versatile molecule with lots of pharmacological properties (Figure 1) including antimicrobial, antioxidant, anticancer, neurological, cardiovascular, hepatoprotective, antiinflammatory, protective of the reproductive system, and anti-obesity agent present in the natural nutrient (Figure 2). The literature available on these properties has been summarized here in this review (Table 1).

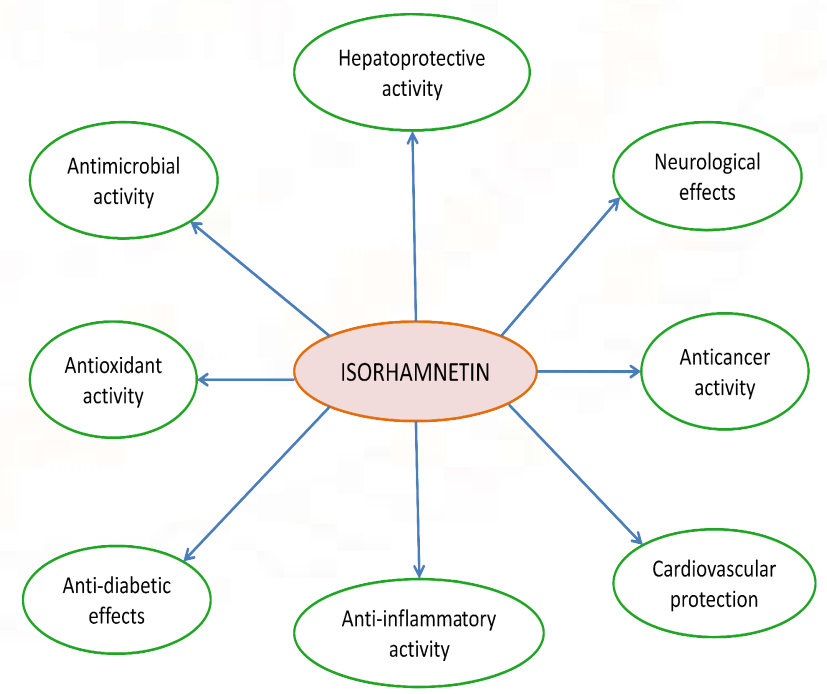

Fig 1: Pharmacological properties of isorhamnetin 


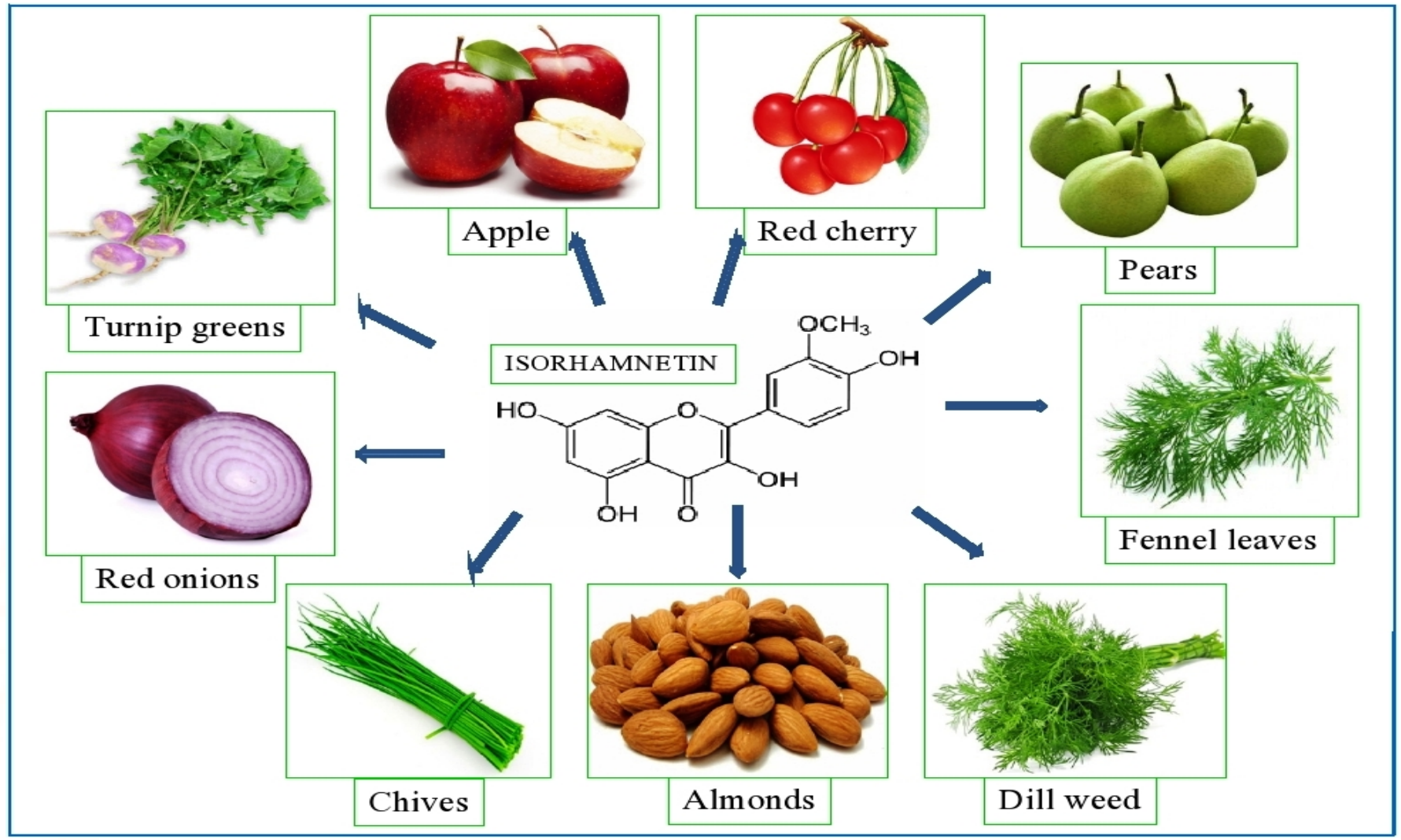

Fig 2: Natural sources and structure of isorhamnetin

\begin{tabular}{|c|c|}
\hline $\begin{array}{c}\text { Pharmacological } \\
\text { property }\end{array}$ & References \\
\hline Antimicrobial activity & {$[16-21]$} \\
\hline Antioxidant activity & {$[22-32]$} \\
\hline $\begin{array}{c}\text { Neurological effects } \\
\text { Anticancer activity } \\
\text { Cardiovascular } \\
\text { protection }\end{array}$ & {$[33-36]$} \\
\hline $\begin{array}{c}\text { Anti-inflammatory } \\
\text { activity }\end{array}$ & {$[31-54]$} \\
\hline $\begin{array}{c}\text { Hepatoprotective } \\
\text { activity }\end{array}$ & {$[38,55-57]$} \\
\hline Anti-diabetic effects & {$[58-60]$} \\
\hline
\end{tabular}

Table 1: Biological activities of isorhamnetin

\section{Antimicrobial activity}

The drug isorhamnetin being a bacteriostatic and it is a good molecule for antibacterial drug research. The compound isorhamnetin extracted from leaf oil of
Ribesnigrum showed a wide range of antimicrobial effect, it's used in the treatment of various bacterial and fungal infections could be beneficial. These inhibitory effects are also interesting in relation to the prevention of contamination in many food products caused by micro-organisms such as Staphylococcus spp., Salmonella spp., Bacillus spp., Pseudomonas fluorescens, and Clostridium botulinum ${ }^{[16]}$. In another study it reveals the reductions in protein and carbohydrate levels following inoculation with Burkholderia cepacia extracts containing isorhamnetin and it compounds as nutrients can make bacterial cell death ${ }^{[17]}$, additionally, it also reported to show anti-influenza effects in vitro and in vivo via direct Haemagglutinin(HA) and Neuraminidase(NA) inhibition, direct or indirect inhibition of the expression of viral HA and NA genes, and suppression of virus-induced autophagy, ROS generation, and ERK phosphorylation ${ }^{[18]}$.

Some of the secondary metabolites including isorhamnetin isolated from $M$. globosumssp. Libanoticums showed an interesting antibacterial activity, which may explain the use of this species in folk medicine to treat skin and urinary tract infections as well as for gastrointestinal diseases ${ }^{[19]}$. 


\section{$>$ Antioxidant activity}

Isorhamnetin has a potential as a natural antioxidant to alternate synthetic substances as food additives ${ }^{[22]}$. Recently, in vivo study, it has been reported that isorhamnetin has antioxidative enzyme activities on the concentrations of cholesterol and lipoperoxide in the serum and liver ${ }^{[23]}$. Likewise isorhamnetin 3 Orobinobioside from Nitrariaretusa leaves enhance antioxidant and antigenotoxic activity in human chronic myelogenous leukemia cell line K562 ${ }^{[29]}$. Moreover, isorhamnetin studied first time of antioxidant activity against Sprague dawley rats liver mitochondria lipid peroxidation induced by $\mathrm{Fe} 2+/$ $\mathrm{Vc}$, from these experimental results demonstrate that isorhamnetin had excellent antioxidant activity ${ }^{[24]}$.

A lot of plant extract studies especially Hippophaer hamnoides ${ }^{[22]}$, Brassica juncea ${ }^{[25]}$, Acacia salicina [26], Ammi visnagaL (Apiaceae) ${ }^{[27]}$, and Brassica juncea $\mathrm{L}{ }^{[28]}$ extracts also has the tremendous antioxidant activity against streptozotocin-induced diabetic rats.

\section{Neurological effects}

Isorhamnetin is a neuroprotective as well as neurotoxic compound like it has been reported to act as a neuroprotector in diet supplemented with Ginkgo biloba extract have distinguished neuromodulatory effects in vivo and illustrates the utility of genomewide expression monitoring to investigate the biological actions of complex extracts [33]. Isorhamnetin has been reported to show beneficial effects and protection of the brain against ischemic injury in mice ${ }^{[34]}$.

\section{Anticancer activity}

Cancer has been found in sixty different parts of the human body and currently requires new therapeutics for its treatment. Isorhamnetin and its precursor have been reported as a potent anticancer agent during in vitro studies in various cancer cell lines and in vivo studies in rodents especially mice ${ }^{[35]}$. Isorhamnetin has been reported to show valuable effect on cancer mainly it was suppresses colon cancer cell growth through the PI3K-Akt-mTOR pathway ${ }^{[37]}$.During an in vitro study on pure isorhamnetin glycosides or when they are mixed with other phytochemicals in an extract obtained from opuntiaficus-indica, the glycosilation pretentious antiproliferative effect ${ }^{[38]}$. However isorhamnetin has potential anticancer activity from the Hippophaer hamnoides L extract against BEL-7402 cells ${ }^{[39]}$. The mitochondria- cytochrome C-caspase-9 cascade mediates isorhamnetin-induced apoptosis makes it was strong candidate for cancer treatment ${ }^{[40]}$.

However, during in vitro study using human Eca-109 cells, isorhamnetin has been reported to inhibit proliferation in human esophageal squamous carcinoma [41]. In a like manner it has antiproliferation effects against lung cancer cell lines (A549 cells) in the in vivo and in vitro studies ${ }^{[42]}$. Likewise isorhamnetin augments the anti-tumor effect of capeciatbine through the negative regulation of NF$\kappa \mathrm{B}$ signaling cascade in gastric cancer ${ }^{[43]}$ and also isorhamnetin inhibits proliferation and invasion and induces apoptosis through the modulation of peroxisome proliferator-activated receptor $\gamma$ activation pathway in gastric cancer ${ }^{[44]}$. Isorhamnetin has the inhibitory effects on the invasion of human breast carcinoma cells by down regulating the expression and activity of matrix metalloproteinase-2/9 ${ }^{[45]}$.

Moreover isorhamnetin might mediate inhibition of HCT-116 cell growth through the perturbation of cell cycle progression and are consistent with the notion that $\mathrm{G} 2 / \mathrm{M}$ checkpoints could be a conserved target for flavonoids in human colon cancer cells, leading to apoptotic and necrotic death. These antiproliferative, apoptotic, necrotic, and cell cycle effects suggest that isorhamnetin may have clinically significant therapeutic and chemopreventive capabilities [46]. Furthermore studies reveal isorhamnetin 3-Orobinobioside from Nitraria retusa leaves enhance antioxidant and antigenotoxic activity in human chronic myelogenous leukemia cell line K562 ${ }^{[47]}$. It is also suppresses skin cancer through direct inhibition of MEK1 and PI3-K ${ }^{[48]}$.

\section{Cardiovascular protection}

Isorhamnetin prevents endothelial dysfunction, superoxide production, and over expression of p47phox induced by angiotensin II in rat aorta ${ }^{[51]}$. Isorhamnetin has a tremendous protective effects on cardiomyocytes against anoxia/reoxygenation-induced injury is mediated by SIRT1 ${ }^{[52]}$ likewise in vivo and in vitro studies give a sufficient report of isorhamnetin has cardiotoxicity against doxorubicin as an inducer [53] in another case isorhamnetin attenuates atherosclerosis by inhibiting macrophage apoptosis via PI3K/AKT activation and HO-1 induction ${ }^{[54]}$.

\section{$>$ Anti-inflammatory activity}

Isorhamnetin attenuate proinflammatory gene expression in lipopolysaccharide -activated murine 
macrophages as well theNF $\kappa \mathrm{B}$ signal transduction cascade, leads to the activation of the Nrf2 pathway. Isorhamnetin may contribute to a down-regulation of inflammatory gene expression both in cultured cells and in vivo these things are clearly give an antiinflammatory effects if isorhamnetin ${ }^{[55]}$. Isolated isorhamnetin and their co-compounds from the Opuntiaficus-indica have tremendous antiinflammatory effects ${ }^{[38]}$. However, isorhamnetin-3-Ogalactoside and its derivatives are clearly explaining anti inflammatory effect against HMGB1-induced inflammatory responses in both HUVECs and CLPinduced septic mice ${ }^{[56]}$ in the same way isorhamnetin inhibit NF- $\kappa \mathrm{B}$ activation along with their inhibitory effect on iNOS expression and NO production in activated macrophages ${ }^{[57]}$.

\section{Hepatoprotective activity}

Isorhamnetin-3-O-galactoside attenuated the increases of NF- $\kappa \mathrm{B}$ and c-Jun nuclear translocation, while it augmented the nuclear level of Nrf2 that is suggest that isorhamnetin-3-O-galactoside ameliorates $\mathrm{CCl}$ induced hepatic damage by enhancing the antioxidative defense system and reducing the inflammatory signaling pathways ${ }^{[58]}$ and also isorhamnetin 3,7-di-O-glucoside in Brassica campestris $L$ leaves, suppressed increases in the plasma ALT and AST activities of mice with liver injury induced by the injection of carbon tetrachloride [59]. However, isorhamnetin protects against the hepatotoxic effect of AA plusiron, and suggest that the AMPK pathway is involved in the mechanism underlying the beneficial effect of isorhamnetin in the liver ${ }^{[60]}$.

\section{Anti-diabetic effects}

Isorhamnetin-3-O-b -D-glucoside tremendous antidiabetic effect in the crucial study, it was isolated from Salicornia herbacea inhibits rat lens aldose reductase and sorbitol accumulation in streptozotocininduced diabetic rat tissues ${ }^{[61]}$.

\section{CONCLUSION}

Most human beings are exposed to flavonoids daily, and thus, their impact on human health is of relevance. The health effects of flavonoids are, still for the most part unknown. Isorhamnetin are studied for their medicine properties within the recent years. We have mentioned a number of the medicine properties, as well as antimicrobial, antioxidant, neurological effects,

anticancer, cardiovascular protection, anti-

inflammatory, hepatoprotective, and Anti-diabetic effects. However, research revealed on antiinflammatory aspect of isorhamnetin isn't enough for its application in humans. Isorhamnetin are versatile molecules and should be investigated more extensively for their wider applications in human health, together with their therapeutic activities.

\section{REFERENCES}

1) Wattenberg LW. Inhibition of carcinogenesis by minor anutrient constituents of the diet, Proc. Nutr. Soc., 1990; 49:173-183.

2) Mabry TJ, Ulubelen A. Chemistry and utilization of phenylpropanoids including flavonoids, coumarins and ligans, J. Agric. Food Chem., 1980; 28:188-196.

3) Araujo RC, Neves FA, Formagio AS, Kassuya CA, Stefanello ME, Souza VV. et al. Evaluation of the anti-mycobacterium tuberculosis activity and in vivo acute toxicity of Annona sylvatic, BMC Complementary Altern. Med., 2014; 14209.

4) Burda S, Oleszek WJ. Antioxidant and antiradical activities of flavonoids, Agric. Food Chem., 2001; 49:2774-2779.

5) Ramos S. Effects of dietary flavonoids on apoptotic pathways related to cancer chemoprevention, Journal of Nutritional Biochemistry, 2007; 18(7):427-442.

6) Rice-Evans C. Flavonoid antioxidants, Current Medicinal Chemistry., 2001; 8:797-807.

7) Steinberg D, Parthasarathy S, Carew TE, Khoo JC, Witztum JL. Beyond cholesterol. Modifications of low-density lipoprotein that increase its atherogenicity, N Engl J Med., 1989; 320:915-924.

8) Katan MB. Flavonoids and heart disease, Am J Clin Nutr., 1997; 65:1542-1543.

9) Pennington JAJ. Food Compos, Anal., 2002; 15:419-434.

10) Saleh NA, Mansour RM, Markham KR. An acylated isorhamnetin glycoside from Aerva javanica, Phytochemistry, 1990; 29:1344-1345.

11) Park JC, Young HS, Yu YB, Lee JH. Isorhamnetin sulphate from the leaves and stems of Oenanthe javanica in Korea, Planta Med., 1995; 61:377-378. 
12) Sikorska M, Matlawska I. Kaempferol, isorhamnetin and their glycosides in the flowers of Asclepias syriaca L, Acta Pol Pharm., 2001; 58:269-272.

13) Schieber A, Keller P, Streker P, Klaiber I, Carle R. Detection of isorhamnetin glycosides in extracts of apples (Malus domestica cv. "Brettacher") by HPLC-PDA and HPLC-APCIMS/MS, Phytochem Anal., 2002;13:87-94.

14) Boesch-Saadatmandi C. Effect of quercetin and its metabolites isorhamnetin and quercetin-3glucuronide on inflammatory gene expression: role of miR-155, J Nutr Biochem., 2011; 22:293299.

15) Kobori M, Masumoto S, Akimoto $Y$, Oike $H$. Chronic dietary intake of quercetin alleviates hepatic fat accumulation associated with consumption of a Western-style diet in C57/BL6J mice, Mol Nutr Food Res., 2011; 55:530-540.

16) Tatjana S, Katarina S, Mihailo R, Gordana Z, Teodora J, Dragana K. et al. Composition and antimicrobial activity of the essential oil of the leaves of black currant Ribes nigrum L. cultivar Cacanska crna, J serb. chem. soc., 2010; 75(1):35-43.

17) Olusesan O, Joseph Ehimidu Changes in the content of antibacterial isorhamnetin 3-glucoside and quercetin 3'-glucoside following inoculation of onion (Allium cepa L. cv. Red Creole) with pseudomonas cepacia, Physiological and Molecular Plant Pathology, 1990; 37:281-292.

18) Ahmed AD, Hye YC, Young BK, Sang-Goo C. Antiviral effect of methylated flavonol isorhamnetin against influenza, PLoS ONE 10(3): e0121610.

19) Daniela R, Carmen F, Adriana B, Alfredo L, Felice S, Sergio R. et al. Antibacterial activity of flavonoids and phenylpropanoids from Marrubium globosum ssp Libanoticum, Phytother. Res., 2007; 21:395-397.

20) Hum NJ, Dasom J, Min-Cheol J, Eunjung L, Bongwhan J, Sungweon R. et al. Antituberculosis activity of a naturally occurring flavonoid, Isorhamnetin, J. Nat. Prod., 2016; 79:961-969.

21) Gomah N. Antimicrobial activity of Calotropis procera Ait. (Asclepiadaceae) and isolation of four flavonoid glycosides as the active constituents, World J Microbiol Biotechnol., 2013; 29:12551262.

22) Liu P, Deng T, Hou X, Wang J. Antioxidant properties of isolated isorhamnetin from the sea buckthorn marc, Plant Foods Hum Nutr., 2009; 64:141-145.

23) Kiharu I, Masamichi O. Effects of isorhamnetin, rhamnetin, and quercetin on the concentrations of cholesterol and lipoperoxide in the serum and liver and on the blood and liver antioxidative enzyme activities of rats, Bio.lci. Biotech. Biochem., 1995; 59(4):595-601.

24) Airen Z, Yanying Y, Jing L, Binbin X, Xiongying Y, Yan Q. et al. Study on the relation of structure and antioxidant activity of isorhamnetin, quercetin, phloretin, silybin and phloretin isonicotinyl hydrazone, Free Radicals and Antioxidants, 2011; 4:39-47.

25) Takako Y, Hyun YK, Eun C, Jae C, Hae YC. Antioxidant effects of isorhamnetin 3, 7-Di-O-âD-glucopyranoside isolated from mustard leaf (Brassica juncea) in rats with streptozotocininduced diabetes, J. Agric. Food Chem., 2002; 50:5490-5495.

26) Ines B, Ines S, Aicha N, Kita V, Kamel G, Anne $\mathrm{MM}$. et al. Antigenotoxic and antioxidant activities of isorhamnetin 3-O neohesperidoside from Acacia salicina, Drug and Chemical Toxicology, 2009; 32(3): 258-267.

27) Reguia B, Habiba K, Ahmed K, Zahia K, Maurice J. Flavonols and antioxidant activity of Ammi visnaga L. (Apiaceae), Rec. Nat. Prod., 2011; 5(1):52-55.

28) Jae SC, Mee JJ, Hye JP, Hae YC, Sam SK. Further isolation of peroxynitrite and 1,1diphenyl-2-picrylhydrazyl radical scavenging isorhamnetin 7-O-glucoside from the leaves of Brassica juncea L, Arch Pharm Res., 2002; 25(5):625-627.

29) Jihed B, Mohammed BS, Ines S, Kamel G, Leila C. Isorhamnetin 3-O-robinobioside from Nitraria retusa leaves enhance antioxidant and antigenotoxic activity in human chronic myelogenous leukemia cell line K562, Complementary and Alternative Medicine, 2012; 12:135.

30) Malgorzata Z, Artur K, Ewa I, Jaromir B. The flavonoids, quercetin and isorhamnetin 3-Oacylglucosides diminish neutrophil oxidative metabolism and lipid peroxidation, Acta biochemica polonica., 2001; 48(1):183-189.

31) Bing S, Gui-Bo S, Jing X, Rong-Chang C, Xin W, Ying W. et al. Isorhamnetin Inhibits H2O2Induced Activation of the Intrinsic Apoptotic 
Pathway in H9c2 Cardiomyocytes Through Scavenging Reactive Oxygen Species and ERK Inactivation, Journal of Cellular Biochemistry, 2012; 113:473-485.

32) Kyuhwa S, Ji Hye Y, Sang CK, Sae KK, Sung HK, Sang MS. The antioxidant effects of isorhamnetin contribute to inhibit COX-2 expression in response to inflammation: A potential role of HO-1, Inflammation, 2014; 37(3):712-722.

33) Coran $\mathrm{MH}$, Siegfried W, Peter A, Gerald R, Lester P, John JM. et al. The in vivo neuromodulatory effects of the herbal medicine ginkgo biloba, Proc of the Nat Aca of Sci., 2001; 98(12):6577-6580.

34) Jin-Jing Z, Jin-Qing S, Shu-Yi P, Kai W. Treatment with isorhamnetin protects the brain against ischemic injury in mice, Neurochem Res., 2016; 41(8):1939-1948.

35) Dajas F. Life or death: Neuroprotective and anticancer effects of quercetin, J Ethnopharmacol., 2012; 143:383-396.

36) Tae HK, Sae-Kwang K, Jong-Sup B. AntiInflammatory activities of isorhamnetin-3-Ogalactoside against HMGB1-induced inflammatory responses in both HUVECs and CLP-induced septic mice, Journal of Cellular Biochemistry, 2013; 114:336-345.

37) Chuan Li, Xi Yang, Cheng Chen, Shaoxin Cai, Junbo Hu. Isorhamnetin suppresses colon cancer cell growth through the PI3K Akt mTOR pathway, Molecular Medicine Reports, 2014; 9: 935-940.

38) Marilena Antunes-Ricardo, Janet A. GutiérrezUribe, Carlos Martínez-Vitela, Sergio O. SernaSaldívar. Topical anti-inflammatory effects of isorhamnetin glycosides isolated from Opuntia ficus-indica, BioMed Research International, 2015; 10.1155/847320.

39) Bao-song T, Yan-Hua L, Zheng-Tao W, Xin-Yi T, Dong-Zhi W. In vitro anti-tumor activity of isorhamnetin isolated from Hippophae rhamnoides L. against BEL-7402 cells, Pharmacological Research, 2006; 54:186-194.

40) Hyo-Jung L, Hyo-Jeong L, Eun-Ok L, Seong-Gyu K, Hyun-Soo B, Cheol-Ho K. et al. Mitochondriacytochrome C caspase-9 cascade mediates isorhamnetin-induced apoptosis, Cancer Letters, 2008; 270:342-353.

41) Gang M, Chunlei Y, Yi Q, Huaying W, Tongtong $Z$, Najuan $Z$. The flavonoid component isorhamnetin in vitro inhibits proliferation and induces apoptosis in Eca-109 cells, ChemicoBiological Interactions, 2007; 167:153-160.

42) Qiong Li, Fu-Qiang R, Chun-Lei Y, Li-Ming Z, Yan-You L, Jing Xiao. et al. Anti-proliferation effects of isorhamnetin on lung cancer cells in vitro and in vivo, APJCP., 2015; 16(7)3035.

43) Kanjoormana A, Muthu K, Lalitha R, Feng L, Kodappully S, Arunachalam C. et al. Isorhamnetin augments the anti-tumor effect of capeciatbine through the negative regulation of $\mathrm{NF}-\kappa \mathrm{B}$ signaling cascade in gastric cancer, Cancer Letters, 2015; 363:28-36.

44) Lalitha R, Kanjoormana A, Muthu K, Feng Li, Kodappully S, Shireen V. et al. Isorhamnetin inhibits proliferation and invasion and induces apoptosis through the modulation of peroxisome proliferator-activated receptor-activation pathway in gastric cancer, The journal of biological chemistry, 2012; 287(45):38028-38040.

45) Chenglin L, Dan Y. Inhibitory effects of isorhamnetin on the invasion of human breast carcinoma cells by down regulating the expression and activity of matrix metalloproteinase-2/9, Nutrition and Cancer, 2015; 0(0):1-10.

46) Sara J, Sergio L, Lourdes MV, Rocio RA, Ana J, Rocio A. et al. The Flavonol isorhamnetin exhibits cytotoxic effects on human colon cancer cells, J. Agric. Food Chem., 2010; 58:10869-10875.

47) Jihed B, Mohammed BS, Ines S, Kamel G, Leila CG. Isorhamnetin 3-O-robinobioside from Nitraria retusa leaves enhance antioxidant and antigenotoxic activity in human chronic myelogenous leukemia cell line K562, Complementary and Alternative Medicine, 2012; 12:135.

48) Jong-Eun K, Dong-Eun L, Ki Won L, Joe Eun S, Sang Kwon S, Jixia Li. et al. Isorhamnetin suppresses skin Cancer through direct inhibition of MEK1 and PI3-K, Cancer Prev Res (Phila), 2011; 4(4):582-91.

49) Antunes-Ricardo M, Moreno-García BE, Gutiérrez-Uribe JA, Araiz-Hernandez D, Alvarez MM, Serna-Saldivar SO. Induction of apoptosis in colon cancer cells treated with isorhamnetin glycosides from opuntia ficus-indica pads, Plant Foods Hum Nutr., 2014; 69:331-336.

50) Shakir MS, Matthew RY, Yava LJH, Lilia I, Moses OEv, Jennifer W. et al. Chemopreventive activity of plant flavonoid isorhamnetin in colorectal cancer is mediated by oncogenic src 
and b-Catenin, Cancer Res., 2013; 73(17):547384.

51) Manuel S, Federica L, Rocio V, Inmaculada CV, Angel C, Rosario J. et al. Quercetin and isorhamnetin prevent endothelial dysfunction, superoxide production, and overexpression of p47phox induced by angiotensin II in rat aorta1, J. Nutr., 2007; 137:910-915.

52) Liqing H, Huan H, Zhantu L, Dan L, Dong Y, Ming H. Protective effects of isorhamnetin on cardiomyocytes against anoxia/reoxygenationinduced injury is mediated by SIRT1, J Cardiovasc Pharmacol., 2016; 67:526-537.

53) Jing S, Guibo S, Xiangbao M, Hongwei W, Yun L, Meng Q. et al. Isorhamnetin protects against doxorubicin-induced cardiotoxicity in vivo and in vitro, PLoS ONE. 2013; 8(5) 64526

54) Yun L, Guibo S, Xi D, Min W, Meng Q, Yingli Y. et al. Isorhamnetin attenuates atherosclerosis by inhibiting macrophage apoptosis via PI3K/ AKT activation and HO-1 induction, PLoS ONE, 2015; 10(3) 0120259.

55) Christine BS, Agnieszka L, Anika EW, Anna S, Alicja J, Jozef D. et al. Effect of quercetin and its metabolites isorhamnetin and quercetin-3glucuronide on inflammatory gene expression: role of miR-155, Journal of Nutritional Biochemistry, 2011; 22:293-299.

56) Tae HK, Sae-Kwang K, Jong-Sup B. AntiInflammatory activities of isorhamnetin-3-Ogalactoside against HMGB1-induced inflammatory responses in both HUVECs and CLP-induced septic mice, J cell Biochem., 2013; 114:336-345.

57) Mari H, Riina N, Pia V, Marina H, Eeva M. Antiinflammatory effects of flavonoids: Genistein, Kaempferol, Quercetin, and Daidzein inhibit STAT-1 and NF- $\kappa$ B activations, whereas flavone, Isorhamnetin, Naringenin, and Pelargonidin inhibit only $N F-\kappa B$ Activation along with their inhibitory effect on iNOS expression and NO production in activated macrophages, Mediators of inflammation, 2007; 10.1155//45673.

58) Dong-Wook K, Hong-Ik C, Kang-Min K, So-Jin $\mathrm{K}$, Jae-Sue $\mathrm{C}$, Yeong. et al. Isorhamnetin-3-Ogalactoside protects against $\mathrm{CCl} 4$-Induced hepatic injury in mice, Biomol Ther., 2012; 20(4):406412.

59) Kiharu I, Tsuyoshi M, Yuri T, Hideyo S. Comparison of the preventive activity of Isorhamnetin glycosides from atsumi-kabu (Red
Turnip, Brassica, campestris L.) Leaves on carbon tetrachloride-induced liver injury in mice, Biosci. Biotechnol. Biochem., 2008; 72 (3):856-860.

60) Guang-Zhi D, Ju-Hee L, Sung-Hwan K, Ji-Hye Y, Il-Je C, Seung HK. et al. AMPK activation by isorhamnetin protects hepatocytes against oxidative stress and mitochondrial dysfunction, European Journal of Pharmacology, 2014; 740:634-640.

61) Yeon S, Sanghyun, Hye S, Bak-Kwang, Kazuo O, Kuk H. Inhibitory effects of Isorhamnetin-3-O-b D-glucoside from Salicornia herbacea on rat lens aldose reductase and sorbitol accumulation in streptozotocin-induced diabetic rat tissues, Biol. Pharm. Bull, 2005; 28(5):916-918. 